\section{Nous avons tous entendu dire que le sens de rotation du tourbillon au fond d'un lavabo qui se vide est différent selon que l'on observe ce phénomène dans l'hémisphère nord ou sud. Mais tenter vraiment l'expérience est autrement plus instructif - et décevant - que de se contenter de répéter cette histoire... À l'échelle d'un lavabo, la rotation de la Terre se mesure en microns}

par seconde, et aura toutes les chances d'être masquée

par un quelconque mouvement résiduel de l'eau.

\section{Observer sans tricher} ce phénomène bien réel reste néanmoins possible, à condition d'être très minutieux et de disposer d'une expérience extrêmement bien contrôlée [1].

\title{
Un pendule de Foucault fluide
}

Jean Boisson, David Cébron, Frédéric Moisy (moisy@fast.u-psud.fr)

et Pierre-Philippe Cortet

Laboratoire FAST, Université Paris-Sud, UPMC, CNRS UMR 7608, 91405 Orsay

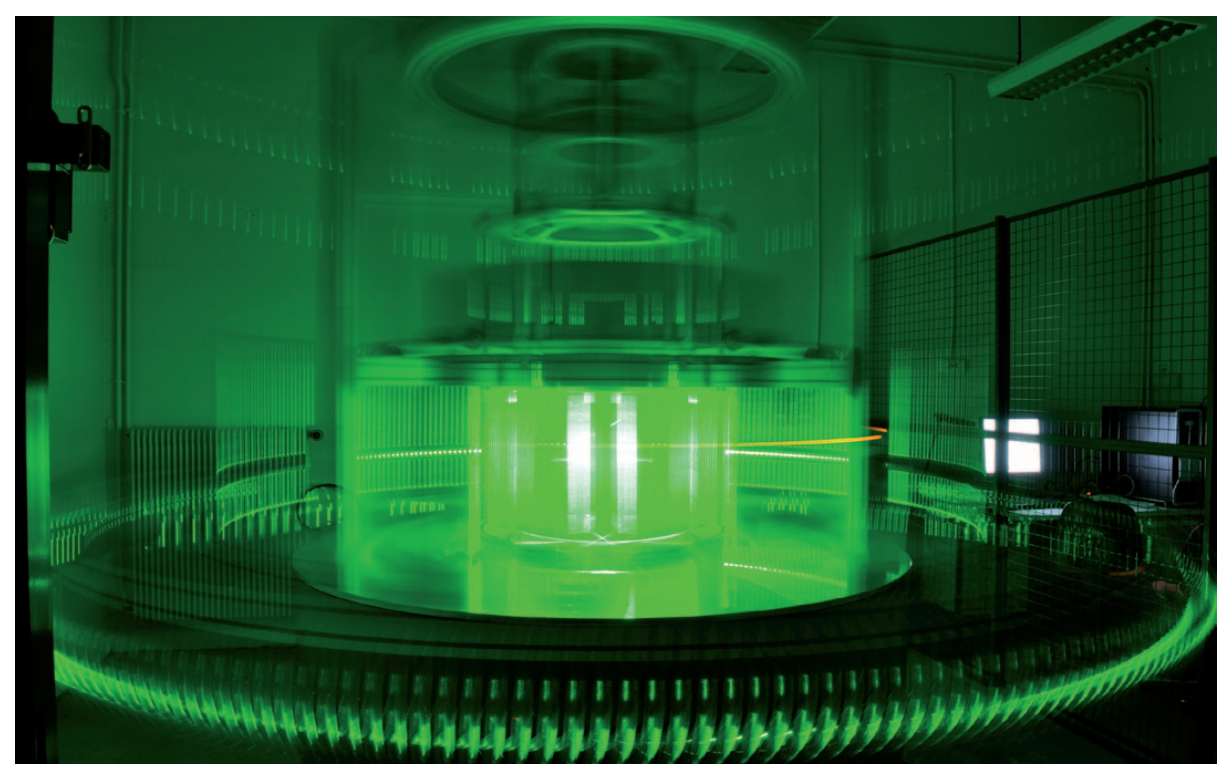

1. La plateforme tournante Gyroflow, installée au laboratoire FAST (Orsay), photographiée ici avec un temps de pause de $10 \mathrm{~s}$. Au centre de la plateforme est disposée une sphère remplie d'eau, mise en rotation à vitesse angulaire $\Omega$. Un système de vélocimétrie par images de particules, embarqué dans le référentiel tournant, permet de mesurer l'écoulement secondaire induit par la rotation de la Terre.

De telles expériences de mécanique des fluides sensibles à la rotation de la Terre ne sont pas fréquentes - on peut les considérer comme des analogues fluides du célèbre pendule de Foucault. L'expérience réalisée récemment au Laboratoire FAST à Orsay est conceptuellement la version la plus simple d'un tel pendule de Foucault fluide [2]. Une sphère en verre d'environ $20 \mathrm{~cm}$ de diamètre, remplie d'eau, est mise en rotation à une vitesse angulaire $\Omega$ constante de quelques tours par minute (fig. 1). Après un régime transitoire de mise en rotation de l'eau par le frottement visqueux entre les couches de fluide (de l'ordre d'une dizaine de minutes [3]), on s'attend naturellement à ce que l'eau tourne exactement à la vitesse angulaire de la sphère. Mais, surprise : même après plusieurs heures, l'eau refuse de tourner à la bonne vitesse ! On détecte dans le référentiel tournant à la vitesse $\Omega$ la présence d'un écoulement secondaire très faible, de l'ordre de quelques dizaines de microns par seconde (fig. 2). Il s'agit en fait d'un tourbillon résiduel d'axe horizontal, fixe dans le référentiel du laboratoire (approximativement orienté selon une ligne Est-Ouest), qui se superpose à l'écoulement de rotation principal imposé par la sphère.

Cet écoulement secondaire a bien pour origine la rotation de la Terre. En effet celle-ci, en imprimant un lent mouvement de précession à l'axe de rotation de la sphère, induit un couple gyroscopique qui défléchit légèrement la trajectoire circulaire du fluide. Ainsi, ce n'est pas directement la vitesse de la Terre qui est détectée, mais un écoulement secondaire résultant de l'équilibre entre ce couple gyroscopique et la friction visqueuse avec la paroi de la sphère. Au final, le fluide tourne autour d'un axe de rotation légèrement incliné par rapport à celui de la sphère, d'un angle de l'ordre de 0,1 degré. Une telle différence aurait évidemment été trop faible pour être mesurée directement depuis le référentiel du laboratoire. Mais, dans le référentiel tournant, la rotation principale se trouve naturellement soustraite, et la différence entre ces deux composantes de rotation apparait sous forme d'un tourbillon horizontal résiduel d'axe fixe par rapport au laboratoire. 
De façon remarquable, de tels écoulements induits par précession sont omniprésents dans le noyau liquide des planètes, ainsi que dans les océans piégés sous des calottes de glace que l'on rencontre dans certains satellites. Par exemple, l'axe de rotation de la Terre, incliné d'environ 23 degrés par rapport au plan de l'écliptique, effectue lui-même un mouvement de précession autour d'un axe fixe avec une période d'environ 26000 ans (précession des équinoxes). Il en résulte un écoulement secondaire dans le noyau liquide, analogue à celui observé dans l'expérience. L'influence de tels mécanismes sur la dynamique du noyau, notamment dans la génération et l'évolution du champ magnétique terrestre, fait l'objet aujourd'hui d'importants débats. Ainsi, cette simple expérience fournit un modèle réduit saisissant des écoulements complexes présents en géophysique et en astrophysique !

\section{Références}

1 A.H. Shapiro, “Bath-tub vortex”, Nature 196 (1962), 1080.

2• J. Boisson et al., "Earth rotation prevents exact solid body rotation of fluids in the laboratory", EPL $\mathbf{9 8}$ (2012), 59002.

3• É. Guyon et al., Hydrodynamique Physique, EDP Sciences (2001).
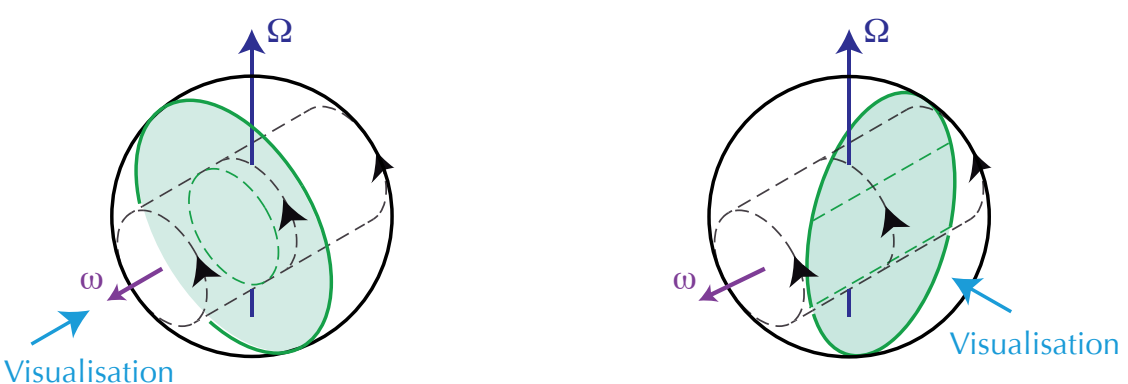

a
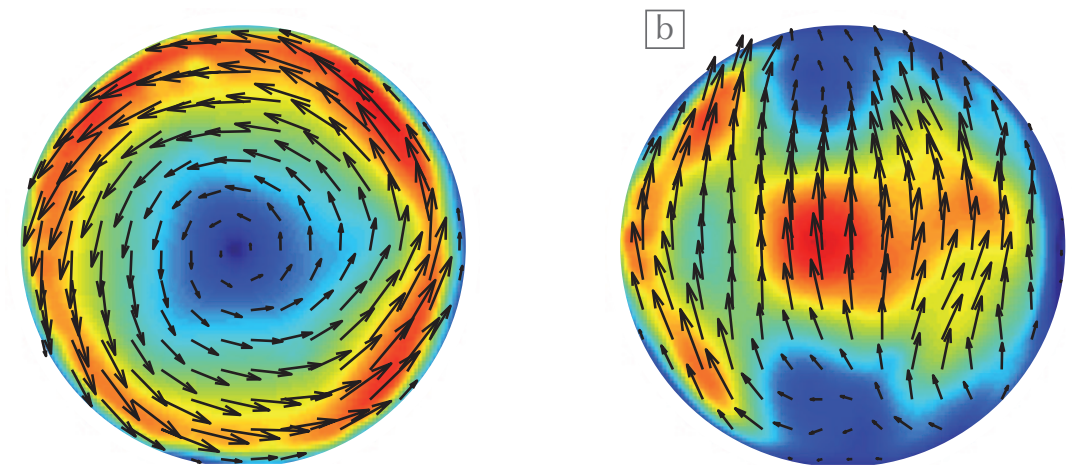

0

$|\mathbf{u}|(\mathrm{m} / \mathrm{s})$

50

2. Visualisation de l'écoulement induit par la rotation de la Terre, mesuré par vélocimétrie par images de particules dans un plan vertical dans le référentiel tournant. Les schémas du haut montrent la sphère qui tourne autour de l'axe $\boldsymbol{\Omega}$ (en indigo), tandis que l'écoulement secondaire induit par précession est un tourbillon horizontal d'axe $\omega$ (en violet), fixe dans le référentiel du laboratoire. Le plan de mesure est indiqué en vert. Les images du bas montrent deux vues instantanées de ce tourbillon secondaire, autour duquel tourne le dispositif de mesure (les flèches et la couleur montrent la direction et le module de la vitesse locale du fluide).

(a) Vue de face, montrant une rotation dans le sens antihoraire.

(b) Vue de côté, montrant un écoulement montant (le plan de mesure est décalé par rapport à l'axe de rotation). 